'To start immune therapy or not? An unusual presentation of longitudinally extensive

$\underline{\text { transverse myelitis with pyrexia' }}$

E. Rounis ${ }^{* 1,2}$, M. I. Leite ${ }^{1,2}$, P.M. Pretorius ${ }^{1,3}$, A. Sen ${ }^{1,2}$

* Corresponding author

1. Neurology and 3. Neuroradiology Departments, John Radcliffe Hospital, Oxford University Hospitals NHS Foundation Trust, United Kingdom

2. Nuffield Department of Clinical Neurosciences, University of Oxford, United Kingdom

E. Rounis, MBBS, PhD, MRCP SCE (Neurol)

M.I. Leite, MD, DPhil

P. Pretorius, MBChB, MSc, FRCR

A. Sen, PhD, FRCP

Keywords: Transverse myelitis, Neuro-myelitis optica spectrum disorders, MOG-antibodies 
Dear Editors,

We present the case of a patient with rapidly progressive transverse myelitis and fevers.

A 26-year-old man, with no recent history of illnesses or foreign travel, was transferred to the John Radcliffe Hospital in Oxford with a 5-day history of bladder disturbance and loss of perineal sensation, followed by ascending sensorimotor deficits, beginning in his legs and spreading to his arms. Around 48 to 72 hours after symptom onset he developed fevers and became tachycardic. Neurological examination revealed increased tone in both legs with bilateral ankle clonus. He had pyramidal-pattern lower limb weakness. Reflexes were brisk throughout with crossed adductor jerks and extensor plantar responses. There was a sensory level to pin prick at T8. Vibration sense was reduced to the knees and joint position sense to the ankles bilaterally. He required catheterization after a bladder scan revealed a $500 \mathrm{ml}$ post-void residual.

During his admission, his temperature rose to more than $39^{\circ} \mathrm{C}$ and was associated with symptoms of rigors and tachycardia. Blood tests revealed an elevated white cell count $\left(22^{*} 10^{\wedge} 9 / \mathrm{L}\right)$ with a neutrophilia $\left(18^{*} 10^{\wedge} 9\right)$ and a CRP of $100 \mathrm{mg}$ per L. The patient had a series of blood cultures which did not grow any organisms. TB cultures from serum and Elispot IGRA were also negative. HIV, mycoplasma, borrelia and syphilis serology were all negative. His ANA, beta2 microglobulin, rheumatoid factor, complement levels and serum angiotensin converting enzyme were normal or negative.

Initial cerebrospinal fluid (CSF) analysis revealed a white cell count of $379^{*} 10^{\wedge} 6(90 \%$ lymphocytes, $10 \%$ polymorphs; there were $356^{*} 10^{\wedge} 6$ red cells). No organisms were grown. The CSF protein was marginally elevated at $600 \mathrm{mg} / \mathrm{L}$. CSF glucose was normal. Oligoclonal IgG bands were paired in CSF and serum. Histopathological analysis of his CSF revealed a lymphocytic inflammatory pleocytosis. TB Elispot and TB CSF PCR) were subsequently 
negative. Flow cytometry was normal. A CT scan of his chest, abdomen and pelvis was also normal.

The patient was treated with intravenous meropenem, ceftriaxone and acyclovir for a presumed systemic or central nervous system infection, whilst waiting for the results of his investigations. However, his fevers did not improve. After completing a course of antibiotics and with negative cultures and PCR for Herpes Simplex Virus, his antimicrobials and antivirals were stopped. His inflammatory markers normalized, but, nonetheless, he continued to experience a swinging pyrexia (Figure 1).

An initial unenhanced MRI scan of his whole spine, demonstrated a longitudinally extensive transverse myelitis (LETM; Figure 1a). A, follow-up MRI of his brain and spine with gadolinium enhancement was performed three days later. This showed florid, patchy leptomeningeal enhancement along the cerebellar folia bilaterally. However, there was no leptomeningeal enhancement or parenchymal enhancement of the spinal cord.

Antibody-testing demonstrated negative aquaporin 4, but positive anti-myelin oligodendrocyte glycoprotein (MOG) antibodies in both serum and CSF, using an immunofluorescence assay previously described by our group [1]. He was initiated on immune therapy with intravenous immunoglobulins (Ivlg). His temperature rapidly lysed and did not return (Figure 1b). Treatment with Ivlg was followed by a course of intravenous methylprednisolone followed by an oral taper of prednisolone. The patient's limb weakness and spasticity improved such that he was able to mobilize unaided and was discharged home with intermittent self-catheterization within one month of his original admission. He remained clinically stable when he was reviewed 3 months later and a repeat of his MOG Ab testing in the serum revealed this was now only borderline positive [1]. 
Traditionally, severe, progressive transverse myelitis is associated with a guarded prognosis. In particular, transverse myelitis associated with fever and a very high white cell count in the CSF suggests an infective etiology, principally tuberculosis. TB myelitis is rare, accounting for less than $1 \%$ of systemic TB cases [2]. Fever, paraplegia and bladder or bowel symptoms are the most common presentations of this condition. In this case the brain MRI appearances were equivocal, and might have suggested an infective cause. However the appearances of the patient's MRI Spine both on unenhanced and gadolinium-enhanced sequences 3 days apart, were somewhat inconsistent with TB myelitis (Figure 1a), in that there were no imaging features of spondylodiscitis, no effacement or compartmentalization of the spinal CSF space and no spinal leptomeningeal enhancement or parenchymal enhancement of the cord. It is noteworthy that recent imaging characterization of MOG-antibody NMOSD have described leptomeningeal enhancement [3].

The case presented here demonstrates a common problem encountered in clinical practice, where an autoimmune disease can very closely mimic infective myelitis. In particular the patient presented here had a swinging fever, an equivocal MRI brain scan and very abnormal CSF cell counts, findings which can lead to a dilemma in initiating immune therapy as such treatment may exacerbate an underlying infection. More rapidly available assays to exclude the latter are of paramount importance. However, early initiation of immunomodulation with Ivlg in the context of infection could perhaps be considered in this context as this is unlikely to cause harm - Ivlg being used as a substitute therapy in some forms of immunodeficiency [4].

Anti-MOG antibodies have relatively recently been described in cases of neuromyelitis optica spectrum disorder (NMOSD) with negative Aquaporin 4 Antibodies. Patients with this disorder comprise up to $20-30 \%$ of AQ4 Ab negative diseases [5-6]. Recent case series have identified approximately $13 \%$ of MOG+ve NMO patients present with transverse myelitis in 
the absence of optic neuritis [7-8] and CSF pleocytosis has been recognized and reported in approximately $70 \%$ of patients [7-9], highlighting its heterogeneity [11]. The acute treatment for this condition is with immunomodulatory therapy, and clinical experience suggests that using a prolonged oral steroid tapering may prevent early relapses [9-11]. As in our patient recovery after immunosuppression can be very good with sphincter disturbance as the predominant residual feature, although for our patient this too completely resolved [11].

While we cannot definitively conclude that there was no underlying initial infection that triggered the neuroinflammatory episode, the lysis of fever with immunomodulatory treatment perhaps suggests that the pyrexia was driven or at least sustained by the underlying anti-MOG antibody syndrome.

The case presented here illustrates that high fever and very high CSF lymphocytosis is still compatible with an autoimmune disease in which immunomodulatory treatment can lead to an excellent recovery with low risk of remission, particularly in cases such as ours who have low MOG-Ab titres at follow-up [10-11].

Figure 1

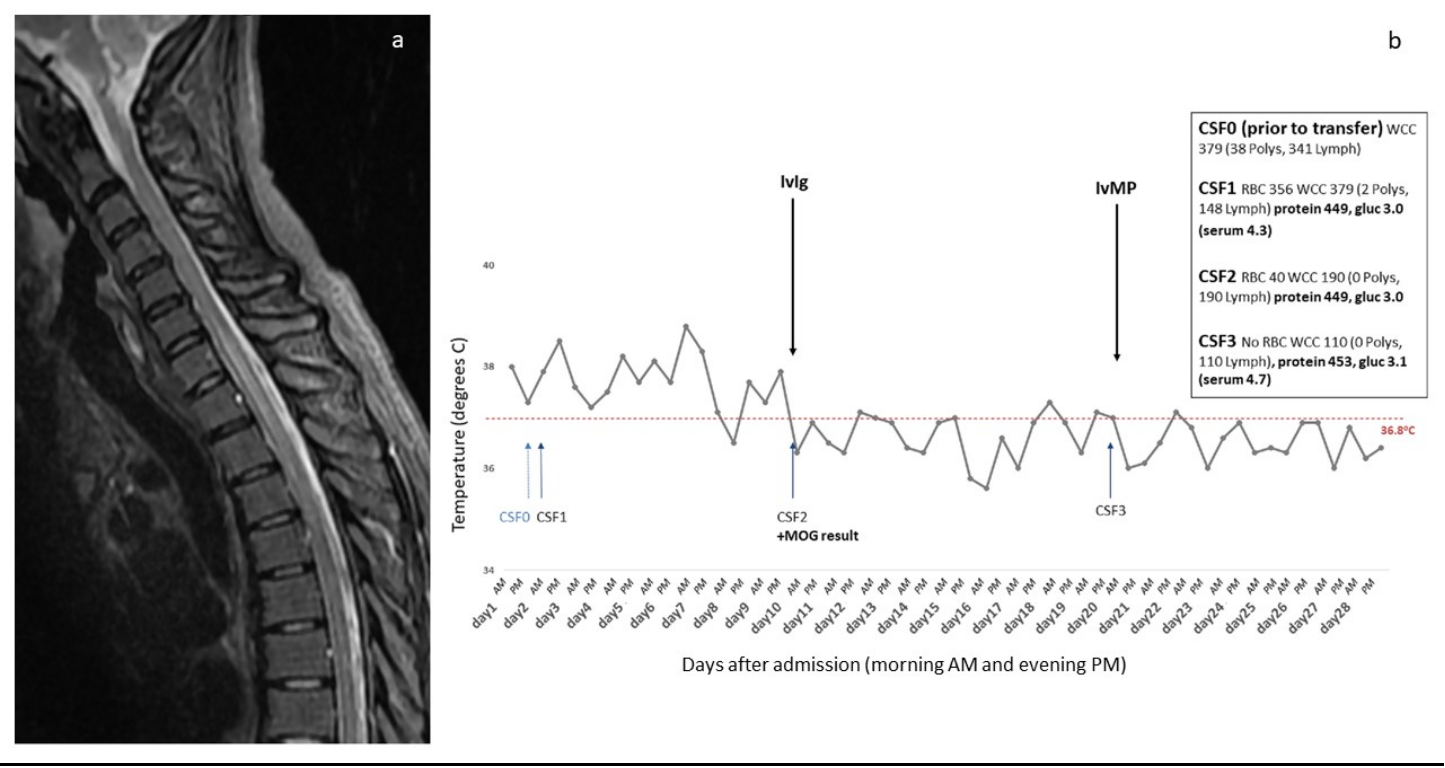

Figure Legends: 
Figure 1a:

Midline sagittal STIR image. There is central T2-hyperintensity associated with slight swelling of the spinal cord. On this image, it is most conspicuous at the C6 to T3 levels but the abnormality extends from approximately C3 to the conus. Gadolinium was not administered for this scan but a gadolinium enhanced MRI scan performed 3 days later did not demonstrate pathological contrast enhancement.

Figure 1b:

Patient's temperature chart with 2 measurements per day over two weeks since date of transfer (day 1). The points at which cerebrospinal fluid (CSF) samples were taken are shown with the blue arrows. The CSF constituents at the time, as well as the ones from a sample collected prior to transfer are reported in the box on the right-hand corner. Glucose and protein are in $\mathrm{mmol} / \mathrm{L}$, cell count is ${ }^{*} 10^{\wedge} 6 / \mathrm{L}$ (Polys: polymorphs, Lymph: lymphocytes). Treatment with intravenous immunoglobulin (IVIg) was started on day 10 (after completion of a full course of antimicrobials that had no effect on temperature), and was supplemented with intravenous Methylprednisolone (IVMP) after serologic confirmation of anti-myelin oligodendrocyte glycoprotein (MOG) antibodies.

\section{Ethical Standards:}

We confirm that we have received the patient's consent in writing for the publication of this case, that there is no identifiable information in this manuscript.

\section{Conflict of Interests:}

M. I. Leite is involved in AQP4 testing; is supported by the National Health Service National Specialised Commissioning Group for Neuromyelitis Optica and by the 
National Institute for Health Research Oxford Biomedical Research Centre; and has received speaking honoraria from Biogen Idec and travel grant from Novartis.

The remaining authors (E.R., P.M.P., A.S.) declare there are no conflicts of interest.

\section{$\underline{\text { References: }}$}

1. Leite MI, Jacob S, Vegas S, Cossins J, Clover L, Morgan BP, Beeson D, Willcox N, Vincent A (2008) IgG1 antibodies to acetylcholine receptors in 'seronegative' myasthenia gravis. Brain 131(pt7) 1940-52.

2. Sahu SK, Giri S, Gupta N (2014) Longitudinal extensive transverse myelitis due to tuberculosis: a report of four cases. Journal of Postgrad Med 60(4): 409-12.

3. Wasay M, Arif H, Khealani B, Ahsan H (2006) Neuroimaging of Tuberculous myelitis. Journal of Neuroimaging 16 (3): 197-205.

4. Mouthon L. and Lortholary O. (2003) Intravenous immunoglobulins in infectious diseases: where do we stand? Clinical Microbiology and Infection. 9(5): 333-338.

5. Jurynczyk M, Geraldes R, Probert F, Woodhall MR, Waters P, Tackley G, DeLuca G, Chandratre S Leite MI, Vincent A, Palace J. (2017) Distinct brain imaging characteristics of autoantibody-mediated CNS conditions and multiple sclerosis. Brain 140 (3): 617-27.

6. Kitley J, Waters P, Woodhall M, Leite MI, Murchison A, George J, Kueker W, Chandratre S, Vincent A, Palace J (2014) Neuromyelitis optica spectrum disorders with aquaporin 4 and myelin-oligodendricyte glycoprotein antibodies: a comparative study. JAMA 71(3): 276-83

7. Sato DK, Callegaro et al (2014) Distinction between MOG Ab positive and AQ4 positive NMO spectrum disorders. Neurology 82(6): 474-481. 
8. Jarius et al. (2016) MOG -lgG in NMO and related disorders: a multicenter study of 50 patients. Part 1: Frequency syndrome specificity, influence of disease activity, long-terms course association with AQP4-IgG and origin. The Journal of Neuroinflammation 13:279.

9. Jarius et al. (2016) MOG-IgG in NMO and related disorders: a multicenter study of 50 patients. Part 2: Epidemiology, clinical presentation, radiological and laboratory features, treatment responses, and long-term outcome. The Journal of Neuroinflammation 13:285.

10. Elovaara I et al. (2008) EFNS guidelines for the use of intravenous immunoglobulins in treatment of neurological diseases. EJN 15 (9): 893-908.

11. Jurynczyk M, Messina S, Woodhall MR, Raza N, Everett R, Roca-Fernandez A, Tackley G, Hamid S, Sheard A, Reynolds G, Chandratre S, Hemingway C, Jacob A, Vincent A, Leite MI, Waters P, Palace J (2017) Clinical presentation and prognosis in MOGantibody disease: a UK study. Brain 140 (12): 3128-38.

\section{Acknowledgements:}

This work was supported by the Oxford NIHR BRC. (A/Prof A. Sen) Dr M.I. Leite is supported by the NMO clinical service at the John Radcliffe Hospital in Oxford. We would like to thank Drs Paddy Waters and Mark R Woodhall for technical support and antibody assay results, as well as Dr S Wilmaratna for referring this gentleman to our care. 